Bei den Aufgaben stellt der Verfasser nicht geringe Anforderungen, bietet abor dadurch viel Anregang.

Die geschichtlichen und literarischen Bemerkungen am Schlusse jedes Abschnittes sind wieder reichhaltig und bei der bekannten Gewissenhaftigkeit des Autors sehr wertroll. Die Figuren sind trotz der Kleinheit übersichtlich, nur bei Fig. 35 (Pohlkes Lehrsatz) wäre eine Vergrößerung vorteilhaft. bemerkt.

Druckfehler habe ich außer den vom Verfasser selbst angefährten nicht

Das nun vollendet vorliegende Werk reiht sich würdig an die großen Gesamtwerke äber Darstellende Geometrie und bringt so viel eigenartiges, daß ich nur wänschen kann, es möge die Würdigung finden, die seinem inneren Werte entspricht.

R. Schüßler.

Technische Übungsaufgaben für darstellende Geometrie. Von Emil Nüller. V. Heft. Leipzig u. Wien. F. Deuticke. 1921. Preis $24 \mathrm{~K}$.

Das Heft enthält in zehn Blättern wieder Beispiele aus den verschiedensten Gebieten, die sich für die konstruktiven Übungen trefflich eignen. Wenn die Blätter richtig ausgenützt werden in der Art, dab bei der Darstellung in irgend einer Projektionsmethode mit Schattenkonstruktion nur einzelne Teile in bedeutender Vergrößerung verwendet werden, so kann man erst richtig beurteilen, wie reicher Übungsstoff in diesen wenigen Blättern enthalten ist. Die Blätter 49 und 49 für kotierte Projektion sind glücklich ausgewählt.

\title{
R. Schüpler.
}

Kurvengeometrie des Baumeisters. Von E. v. M ecenseffy. 6 Hefte mit 168 Abbildungen. Verlag W. Ernst \& Sohn, Berlin., 1920. Preis M. 42.90 ohne Zuschlag:

Wie der Verfasser dieses sehr eingehenden und mit sorgfältigen Figuren versehenen Werkes selbst hërvorhebt, handelt es sich um ein Grenzgebiet zwischen Baukunst und Mathematik, nämlich um gewisse ebene Kurven, deren innere Eigenschaften einerseits dem Architekten wenig geläufig sind, deren eingehende mathematische Behandlang anderseits gerade für die Zwecke der Bankunst nur gelegentlich vorkommt. Das Buch wird den beiden sich daraus erhebenden Forderungen gerecht, wie dies am dentlichsten im 1. Heft zum Ausdruck kommt, wo die "Korbbogen“ behandelt werden. Darunter wird /eine Folge von Kreisbögen verstanden, die eine gewisse theoretisch angenommene Kurve (Kegelschnitt, Zykloide, Cassinische Kurve usw.) ersetzen sollen, wobei sich der Baumeister außer von technischen Gründen vom Urteil über eine möglichst große bildliche Annäherung der Ersatzkarve an die gegebene leiten läßt; aus der Erfahrung heraus gibt der Verfasser auch eine Formel (S. 2) für die Anzahl der ersetzenden Kreisbogenstücke. Am meisten dürfte gerade dieses Heft das Interesse der Geometer erwecken, für die in der letzten Zeit das verwand te Problem der ,graphischen Krümmungskreise" (E. Kruppa, Sitzungsber. Ak. Wien 1918) an Bedeatung gewonnen hat. Das 2. Heft; „Übergänge" betitelt, gibt dem Baumeister die Mittel an die Hand, für Krümmungsübergänge unter gegebenen Bedingungen die geeignetsten Linien zuerst theoretisch kennen und einschalten $\mathrm{zu}$ lernen (was mittels leichtfaßlicher Ausführungen in projektiver 